
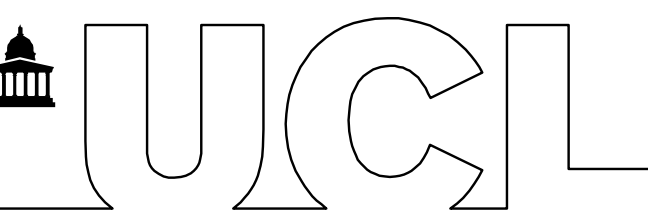

Pegram, T; (2015) Governing Relationships: The New Architecture in Global Human Rights Governance. Millennium - Journal of International Studies, 43 (2) pp. 618-639.

10.1177/0305829814562016. Downloaded from UCL Discovery: $\underline{\text { http://discovery.ucl.ac.uk/1462795 }}$

\title{
ARTICLE
}

\section{Governing Relationships: The New Architecture in Global Human Rights Governance}

\author{
Pegram, Thomas \\ School of Arts and Social Sciences, University College London
}

\section{Introduction}

The international human rights regime has undergone extraordinary expansion in the last thirty years, evident in an increasingly sophisticated framework of treaties, networks, institutions, and ambitious standards. Now widely regarded as a core component of interstate and transnational global affairs, few international relations (IR) scholars anticipated the contemporary reach of human rights. ${ }^{1}$ The transformative potential of an increasingly intrusive human rights architecture should not be underestimated. However, concerns persist regarding the disjuncture between human rights system rules and practices on the ground. This 'compliance gap' is exacerbated by the relative absence of implementation arrangements authorised to enforce a global human rights governance agenda.

The terms of this debate in IR reflect the limitations of a discipline which has only recently extended its gaze beyond interstate relations. IR scholars have struggled to accommodate multiple scales of actors, authority structures, and societal conditions into their explanatory frameworks. As such, human rights scholarship has only lately engaged with key empirical questions on governance in this issue-area. ${ }^{2} \mathrm{~A}$ more problem-oriented scholarship on international organisations (IGOs) and global governance offers a promising point of departure for evaluating the interactions and effects of increasingly dense IGO ecologies in the area of human rights. ${ }^{3}$

Global governance scholarship has begun to explore these trends broadly, using the concept of 'governance architecture', defined as 'the overarching system of public and private institutions that are valid or active in a given issue area in world politics'. ${ }^{4}$ In analyzing the presence or (more often) the absence of integrated global architecture incorporating regimes, institutions and their component agents, structures norms and procedures - scholars have generated significant insights into the impact of institutional complexes, overlaps and interlinkages, on governance outcomes across diverse issue-

\footnotetext{
${ }^{1}$ John Vincent, Human Rights and International Relations (Cambridge: Cambridge University Press, 1986).

2 Par Engstrom, 'The Effectiveness of International and Regional Human Rights Regimes', in Robert A. Denemark (ed.), The International Studies Encyclopaedia (Oxford: Blackwell Publishing, 2010).

${ }^{3}$ Thomas G. Weiss and Rorden Wilkinson, 'Rethinking Global Governance? Complexity, Authority, Power, Change', International Studies Quarterly 58, no. 1 (2014): 207-215.

${ }^{4}$ Frank Biermann et al., 'The Fragmentation of Global Governance Architectures: A Framework for Analysis', Global Environmental Politics 9, no. 4 (2009): 15.
} 
areas. ${ }^{5}$ This scholarship has made important advances in terms of explicating architectures, making legible what may at first glance seem to be quite incoherent and disparate elements. However, much less is known about the actual effects of global architectures, with the jury still out on the relative merits of integration versus fragmentation and, crucially, when and why global structures matter for closing compliance gaps on the ground. ${ }^{6}$

This concern is particularly acute in the domain of global human rights governance. As this study highlights, the global human rights architecture is highly articulated, notable for its profusion of non-state actors and levels of formal linkages across levels of governance. A large rationalist literature on the mechanics of the human rights architecture suggests that differential structural configurations can generate powerful instrumental effects on state behaviour and governance outcomes. ${ }^{7}$ However, other observers caution that such rationalist claims are often not borne out by the evidence. ${ }^{8}$ In particular, they highlight the importance of agency and political contestation in understanding outcomes which deviate from formal models, especially as we engage the question of compliance gaps in domestic jurisdictions. ${ }^{9}$ Taking the issue-area of global human rights governance, this study distinguishes between two central questions: how to best delineate and describe analytically the global actors, processes and structures in this domain and their interactions? When and why does the global human rights architecture actually matter for human rights outcomes? The predominant focus of this article is on the first question, but with a view to exploring the analytical distinction between structure and effects for advancing explanatory research on the efficacy of global structures.

The notion of a governance 'architecture' is adopted here as a heuristic lens capable of accommodating both a rationalist concern for structure and its potential effects on (principally instrumental) behaviour of governance actors, as well as a relationship-based analysis of 'global governors' and the interactional effects of political units within authorising structures. Both accounts provide the coordinates for deepening conceptual and theoretical understandings of global governance. However, both display limitations. The instrumental account tends to privilege structural properties and instrumental rationales focused on resource competition, while screening out agency and political conflict. The relational account, with its emphasis on synergies and tensions among governing actors, while instructive, does not necessarily translate into a specified analytical framework.

This article seeks to specify analytically a governance architecture from the global to the local level within an issue-specific governance domain. A methodological nationalism in much of the IR literature, tending towards arbitrary segmentation of political arenas, has hampered efforts to engage questions of multi-level governance and compliance. ${ }^{10} \mathrm{~A}$ particularly promising advance towards a unified theory of (global) governance can be found

\footnotetext{
${ }^{5}$ David G. Victor and Kal Raustiala, 'The regime complex for plant genetic resources', International Organization 32, no. 2 (2004), 147-54. I follow Stephen Krasner's definition of regimes. See Stephen D. Krasner, International Regimes (Ithaca: Cornell University Press, 1983), 2.

${ }^{6}$ Michael Zürn and Benjamin Faude, 'On Fragmentation, Differentiation, and Coordination', Global Environmental Politics 13, no. 3 (2013), 119-30.

${ }^{7}$ Emilie Hafner-Burton, 'A social science of human rights', Journal of Peace Research 51, no. 2 (2014): 273-286.

${ }^{8}$ Deborah D. Avant et al. (eds.), Who Governs the Globe? (Cambridge: Cambridge University Press, 2010).

${ }_{9}^{9}$ Greg Shaffer, Transnational Legal Ordering and State Change (Cambridge: Cambridge University Press, 2014)

${ }^{10}$ For an instructive discussion see Michael Zürn, 'Global governance as multi-level governance', in Henrik Enderlein et al. (eds.) Handbook on Multi-level Governance (Northampton: Edward Elgar 2010), 80-102.
} 
in the work of Abbott and colleagues on orchestration. ${ }^{11}$ This theory builds upon a large body of work in the field of new governance in public administration and transnationalism to develop a general theory of governance, with application across political units, as well as scales of governance. Distinct from principal-agent models of governance, orchestration applies when a focal actor (the orchestrator) enlists and supports third-party actors (the intermediary) to address the target in pursuit of shared governance objectives. It occurs when three conditions are met: (1) the orchestrator seeks to influence the behaviour of the target indirectly via intermediaries, and (2) the orchestrator does not exercise control over the intermediary, which, in turn, (3) cannot compel compliance of the target. ${ }^{12}$

I use the example of the United Nations torture prevention architecture, as established by the Optional Protocol to the Convention Against Torture (OPCAT), to explore the utility of orchestration theory in generating insight into a new generation of global human rights architecture. ${ }^{13}$ It finds that orchestration provides a powerful toolkit for robust relational specification of actors and linkages across levels of analysis. Beyond this analytical descriptive task, the article also probes the claim that orchestration arrangements can enhance the compliance objectives of the OPCAT architecture: eradicating the practice of torture in domestic jurisdictions. Placing particular emphasis on successful enlistment of local actors into global governance programmes, it explores the premise that orchestration is more likely to secure compliance where governance rule systems are formally articulated in a global architecture and there is goal convergence among political units. I use the former concept to refer to the stabilising properties of structural and normative overlaps, and the latter to evaluate how goal convergence between global governance programmes and domestic pro-compliance actors reinforces or undermines compliance. In so doing, the article further highlights the limitations of an orchestration frame premised on voluntary participation for understanding the actual efficacy of the OPCAT architecture in closing the compliance gap.

Importantly, the article also illustrates an extension of the orchestration arrangement to the domestic level, observing role alternation among orchestrators, intermediaries and targets. This builds upon the Abbott et al. observation that orchestration 'may define the entire chain of governance relationships'. ${ }^{14}$ In contrast to a linear relational model, this article presents four possible dynamics, with important implications for compliance: simple orchestration (goals aligned across political units), competitive orchestration (goals between orchestrator and intermediary aligned, but may compete for finite resources), cascade orchestration (civil society pressure on target to comply, even absent a relationship with the orchestrator), and reverse orchestration (target instrumentalizes the intermediary to influence the orchestrator). This exploratory exercise captures a contemporary climate of dispersed authority, directing our attention away from structure to the close interrelation between material power and coordination capacity of diverse governance actors. Orchestration theory can serve to usefully frame the OPCAT strategic environment and accommodate the possibility of unintended consequences. It offers less insight, however, into those factors which are likely to determine such political outcomes. As outlined here, additional analytical tools are likely required to advance an explanatory agenda of how global arrangements transform the domestic politics of compliance.

\footnotetext{
${ }^{11}$ Abbott et al., International Organizations as Orchestrators (Cambridge: Cambridge University Press, forthcoming), Ch. 1.

${ }^{12}$ Abbott et al., International Organizations as Orchestrators, Ch. 1.

${ }^{13}$ Optional Protocol to the Convention against Torture and other Cruel, Inhuman or Degrading Treatment or Punishment, UNGA Res. A/RES/57/199, 18 December 2002. Entered into force on 22 June 2006.

${ }^{14}$ Abbott et al., International Organizations as Orchestrators, Ch. 14.
} 
This article begins by evaluating the idea of global human rights governance and architecture, before turning to the application of orchestration theory. Using the torture prevention regime as an example, it outlines how articulation of global architecture has begun to reconfigure relationships among key governance actors. It explores the interaction between two core political units (orchestrator and intermediary), with a focus on formal articulation of their governing relationship as well as goal convergence. The potential implications for the efficacy of the OPCAT architecture are explored. The article concludes by highlighting what the analysis means for human rights and global governance research more generally.

\section{Bridging gaps: global human rights governance}

Global human rights governance bears the hallmarks of Rosenau's dictum on 'governance without government', ${ }^{15}$ displaying multiplying forms and structures of authority, regime complexity, actor proliferation and, importantly, the capacity for transformation. States remain prominent within human rights governance. However, the exercise and effects of state power in this domain are undergoing significant change, informed by emergent forms of private and hybrid authority. This trend has been documented in a large body of interdisciplinary work offering micro and macro accounts of the origins of international human rights regimes and the gradual ceding of delegated authority by state parties to independent structures and agents at both the domestic and global level. ${ }^{16}$

However, often disparate actors, arenas and mechanisms of influence have been considered in isolation or as dichotomous. This is symptomatic of a more general trend in IR which has tended to isolate component parts of the global governance whole. The importance of organisational environment has frequently been bracketed outside the analytical frame or disregarded altogether. It is only recently that scholars have begun to apply a broader view to global governance phenomena. In part, this is a response to denser organisational ecologies and functional complexity at the international level, combined with growing uncertainty around causal relationships. Scholars have employed a range of exploratory conceptual frameworks to open up exploration of system-level trends across global governance arenas. ${ }^{17}$

Our understanding of how the human rights organisational environment 'hangs together' remains under-specified, with potentially important implications also for understanding the efficacy of global structures. A lively prescriptive debate has focused on the overall influence of the international human rights regime, with studies arriving at conflicting conclusions. ${ }^{18}$ One possibility is that conflicting patterns stem more from a paucity of data and limited understanding of the many steps which separate international instruments from practices on the ground than from any methodological proclivities. Making legible the governance ontological whole beyond the state is therefore particularly important. As David Lake has written, '[t]he distinction between domestic and international politics as now construed is untenable...We ought to be seeking a unified theory of governance, not artificially segmenting realms of politics by arbitrary assumptions'. ${ }^{19}$

\footnotetext{
15 James Rosenau, 'Governance in the Twenty-first Century', Global Governance 1, no. 1 (1995): 13.

${ }^{16}$ See, for example, Andrew Moravcsik, 'The Origins of Human Rights Regimes: Democratic Delegation in Post War Europe', International Organization 54, no. 2 (2000): 217-252; Margaret Keck and Kathryn Sikkink, Activists Beyond Borders (Ithaca: Cornell University Press, 1998).

${ }^{17}$ John Ruggie, 'Global Governance and "New Governance Theory": Lessons from Business and Human Rights', Global Governance 20, no. 1 (2014): 5-17.

${ }^{18}$ Beth Simmons, 'Treaty Compliance and Violation', Annual Review of Political Science 13 (2010): 273-296.

${ }^{19}$ David Lake, 'Rightful rules: Authority, order and the foundations of global governance', International Studies Quarterly 54, no. 3 (2010): 608.
} 
As many authors have remarked, human rights unlike international trade and security, do not privilege the interests of the authorising principal, but rather the individuals at risk of abuse by those same contracting principals. ${ }^{20}$ As such, traditional interstate mechanisms that explain compliance in other fields, such as reciprocity and retaliation, have limited application. Realist scholars have dismissed human rights as merely an artifice of powerful state interests. However, an empirically-driven research agenda on international diffusion and domestic mobilisation effects has decisively problematised such claims. ${ }^{21}$ The general absence of interstate enforcement has opened up fruitful, methodologically plural inquiry into a diverse range of regulatory logics and identification of new authority structures at the substate, domestic and transnational level. ${ }^{22}$ Explaining why some international human rights norms are effective in some contexts but not in others has proven more problematic for rationalist frameworks. Some scholars have turned to theories of transnational and domestic pro-compliance mobilisation and political contestation for answers. ${ }^{23}$ This is a necessary step towards reasserting the close interrelation between material power and the coordination capacity of non-state actors, an issue often neglected by constructivists. Another strand of research highlights the range of delegated authority within IGO structures and the ability of sub-state and IGO entrepreneurs to pursue governance goals at the limits of, or beyond, state agreement. ${ }^{24}$

In effect, we are witnessing a dispersion of authority at all levels of human rights governance. However, our understanding of the analytically distinct but related issues of how political units are integrated and interact within the global whole, and, crucially, with what effect, remains partial. To overcome this explanatory deficit, it is necessary to reassert the presence of politics and power in global human rights governance. As Avant et al. note, scholars 'rarely talk about global governors'. ${ }^{25}$ We might add that scholars rarely talk about domestic politics and power. Global human rights governance is a particularly instructive realm in which to engage such concerns, given its radical intent as a 'power mediator': whereby it seeks to empower materially weak actors in asymmetric power relationships irrespective of territorial boundaries. ${ }^{26}$ This is not the place for extended conceptual discussion of how a global governance conceptualisation may encapsulate such a radical humanist project. Suffice to say, we must be awake to the potential objectification of 'global governance' as a mask for ideological agendas. ${ }^{27}$ In this sense, a further pending empirical task involves inquiring into global human rights architecture and the key political question: How are these regulatory governance arrangements connected to power structures: whose interests are being protected, and whose values promoted?

\subsection{Conceptualising global human rights architecture}

What is 'global' in global human rights architecture? Beyond the existence of an institutional apparatus above the nation state, Zürn advances two additional conditions as representative of global governance: (1) the global level must be autonomous, with evidence of delegation of power to realms outside state structures (or, more specifically, national executives), and (2) the global level must form part of an interactive system, as opposed to a segmented level

\footnotetext{
${ }^{20}$ Moravscik, 'The Origins of Human Rights Regimes'.

${ }^{21}$ Emilie Hafner-Burton, 'A social science of human rights', Journal of Peace Research 51, no. 2 (2014): 273-286.

${ }_{22}$ See Thomas Risse et al. (eds.), The Persistent Power of Human Rights (Cambridge: Cambridge University Press, 2013).

${ }^{23}$ Alison Brysk, Speaking Rights to Power (Oxford: Oxford University Press, 2013).

${ }^{24}$ Abraham L. Newman, 'International organization control under conditions of dual delegation', in Deborah D. Avant et al., Who Governs the Globe?, 131-152.

${ }^{25}$ Avant et al., Who Governs the Globe, 1.

${ }^{26}$ Christian Reus-Smit, 'Human rights in a global ecumene', International Affairs 87, no. 5 (2011): 1210.

${ }^{27}$ Iver B. Neumann and Ole Sending, Governing the Global Polity (Michigan: University of Michigan Press, 2010).
} 
operating independent of the whole. ${ }^{28}$ There must be, in Zürn's words, some indication of 'functional' or 'stratified' differentiation. An expansive human rights architecture meets both criteria, with significant governance functions (rule-making, stewardship, advocacy, accountability, monitoring, and coordination) delegated to a range of authorities, from IGOs, to civil society and sub-state units. However, it is material to note that states (or more accurately, certain governmental actors) retain significant control over core governance functions, the most visible of which are resource allocation and implementation. In turn, articulation of actors and functions along linked chains of governance is evident.

The international human rights regime is relatively integrated, with an apex rule-making body, the Human Rights Council (HRC), located in Geneva. This body is supported by the UN Office of the High Commissioner for Human Rights $(\mathrm{OHCHR})$, as well as a phalanx of auxiliary bureaucratic structures and agencies. Much of this international machinery has emerged or been strengthened since the early 1990s in the wake of an operational shift towards implementation at the national level. ${ }^{29}$ In turn, there has been significant expansion of regional-level rule-making regimes, most notably in Europe and Latin America, but also emergent in Africa and the Asia-Pacific. ${ }^{30}$ However, governance relationships between the global and the local societal level have remained largely segmented, mediated by state authority and a selective group of transnational actors. As Zürn notes, there is a pressing need 'to legitimate decisions more directly in order to make the two-step authority relationship viable'. ${ }^{31}$ The new OPCAT architecture described below represents an organisational response to this major deficit.

A focus on architecture to explore global human rights governance is intuitively appealing. The language of design provides a reassuring sense of structure to often highly decentralised, loosely coupled realms of politics. From a functionalist perspective, architecture provides a platform upon which to build more effective and efficient governance arrangements. Guided by the concepts of subsidiarity (decisions should be made at as local a level as possible) and irreducibility (certain problems can only be addressed at the global level), rationalist scholarship emphasises the importance of limiting negative externalities of decentralised action, efficiency gains through coordination, and functional differentiation. ${ }^{32}$ To achieve these objectives, they advocate effective management of participating states through the employment of material incentives and sanctions to reduce transaction costs and secure credible commitments. However, too often, rationalists reify interests in their explanatory frameworks and underplay or neglect altogether the role of legitimacy in determining the preferences of political units within authorizing structures. ${ }^{33}$

Observing legitimacy mechanisms poses a methodological challenge. Nevertheless, given the relative absence of hierarchical control at the international level, coupled with prevalent democratic deficits, legitimate authority may be the crucial additive to achieving cooperation and regulation. An alternative critique of architecture questions the attempt to render social relationships as 'permanent, functional, and indeed perhaps beyond rational reproach' ${ }^{34}$ It is important not to exaggerate claims of interlocking global, regional or local structures. In practice, there are few examples of (effective) apex institutions in global human rights.

\footnotetext{
${ }^{28}$ Zürn, 'Global governance as multi-level governance': 81 .

${ }^{29}$ See UN General Assembly Res. 46/124, 17 December 1991.

${ }^{30}$ Engstrom, 'The Effectiveness of International and Regional Human Rights Regimes'.

${ }^{31}$ Zürn, 'Global governance as multi-level governance', 92.

${ }^{32}$ James Fearon, 'Bargaining, Enforcement, and International Cooperation', International Organization 52, no. 2 (1998): 269-305.

${ }^{33}$ Robert Keohane, 'Governance in a Partially Globalized World', American Political Science Review 95, no. 1 (2001): 1-13.

${ }^{34}$ Laurence Whitehead, 'Of Bubbles and Buildings: Financial Architecture in a Liberal Democratic Era', in Armijo, Debating the Global Financial Architecture, 283.
} 
Authority is routinely contested and formal articulation of rule systems in structure remains emergent. In an explicitly normative governance regime such as human rights, this raises important questions of scope and boundary. Who are the legitimate stewards? Where does their scope of action begin and end, and what is the basis of their authority? Any durable human rights architecture must be based not only on procedural obligation, but also on the conflictual politics of authority among both the governors and the governed.

A more precise use of the architecture concept can illuminate the structures and political units which serve as both venues for decision-making and agents for action within multiactor governance systems. This contrasts with Biermann et al.'s expansive view of architecture as the 'meta-level of governance', which incorporates within its purview multiple regimes, institutions and all of their component elements. ${ }^{35}$ The approach adopted here reflects an interest in magnifying how governance rule-systems are purposively articulated within issue-specific domains. Architecture reflects not just legal form but also normative and political understandings and expectations. Rather than simply being the aggregation of formal rules and structure, it is also composed of political units with diverse interests. Close attention must therefore be given to how these political units are integrated. As Mugge notes, 'assessments of legitimacy have to focus on the actual workings of institutions, not on formal flows of authority, information and accountability'. ${ }^{36}$

This insight raises the empirical question: to what extent can particular configurations of global architecture actually enable (or disable) pro-compliance actors to advance their governance objectives? Put even more bluntly, to what effect do global rule frameworks actually influence compliance outcomes? In this regard, analysts may focus on formal safeguards of independence, representation, and equity. On a normative level, the question speaks to a fundamental paradox in the human rights enterprise: principal moral hazard. As Rennger cautions, 'it is an obvious fact that in order to [advance the human rights agenda] we have to give states and other agents more power, not less'. ${ }^{37}$ However, in light of this fact, it is also important to acknowledge that states are not unitary actors, but are made up of multiple sub-state units whose preferences may deviate from those of government. This is particularly apparent in global human rights governance where sub-state units are often subject to dual delegation, whereby states have simultaneously delegated to international organisations and to sub-state actors. ${ }^{38}$ As such, a key task for analysts is to evaluate preference ordering among sub-state units within global human rights governance chains and goal convergence among functionally differentiated governance actors.

Architecture further conveys the notion of normative standards imprinted onto actual structures and, more controversially, practices. The articulation of global governance structures to the local level encapsulates ongoing efforts to 'work human rights into the cracks of international society'. ${ }^{39}$ From a functionalist perspective, it marks a shift from rulemaking to rule-implementation, with the ex-post costs of rule recognition raised through the installation of dedicated agents with sufficient autonomy and power to advance global rule systems locally. It also reflects a differentiation of authority and functions, extending from collective principals (states) to first, second and potentially multiple-tier governance agents. This may have significant implications for both the legitimacy and performance of governance architecture. Additional locations for coordination of policies may enhance performance. However, as global governance chains become extended to domestic

\footnotetext{
${ }^{35}$ Biermann et al., 'The Fragmentation': 15-16.

${ }^{36}$ Daniel Mugge, 'Limits of Legitimacy and the Primacy of Politics in Financial Governance', Review of International Political Economy 18, no. 1 (2011): 54.

37 Nicholas Rennger, 'The world turned upside down? Human Rights and International Relations', International Affairs 87, no. 5 (2011): 1177.

${ }^{38}$ Newman, 'International organization control under conditions of dual delegation'.

${ }^{39}$ Vincent, Human Rights and International Relations, 3.
} 
jurisdictions, questions of legitimate authority, representation, and political contestation are likely to intervene. In the absence of a direct relationship between global structures and the intended beneficiaries (or governed), architecture which does not display a capacity to accommodate political contestation is unlikely to achieve the desired outcome. ${ }^{40}$

This is well-illustrated by interrogating a prevalent assumption in the functionalist literature regarding informational effects of international instruments at the domestic level. ${ }^{41}$ More information is assumed to enhance coordination. However, less attention is generally paid to those conditions under which information becomes powerful. As Johnston puts it, there is a potential 'infinite regress problem' in establishing the credibility of information at or in any given place. ${ }^{42}$ Explaining the local impact of information necessitates attention not only to coordination, but also to the legitimate authority and power of the messenger(s) and its distributional implications for competing domestic political forces. The ability of global governors to steward governance processes in local contexts, especially those defined by high levels of rule contestation, will be reliant upon convergence between the objectives of global governors and those of domestic pro-compliance supporting coalitions willing and able to engage in the often costly (sometimes dangerous) politics of social change. Evaluating the relationship between political units within global governance architectures is therefore essential to understanding outcomes.

\section{Orchestrating global human rights governance}

In order to generate insight into the interplay of political units within a multi-scale governance architecture, this article employs orchestration theory. ${ }^{43}$ Orchestration can be defined as when a governance actor (the orchestrator) enlists and supports third-party actors (the intermediary) to address (the target) indirectly in pursuit of shared governance objectives. As a descriptive analytical theory, it displays a number of general application merits, specifying types of actors, their governance relationships, and the scope for governance mobilisation in light of prevailing problem structures and strategic environments. It is important, however, not to conflate opportunity space under conditions of orchestration with compliance action on the part of the agent. As developed here, orchestration theory represents an important advance on formalist scholarship in terms of theorising global governance domains as strategic social environments. However, it may offer less explanatory insight into the actual compliance effects of global governance programmes on the ground.

Orchestration theory responds to system-level trends in global governance which mirror, to some extent, the new governance model of regulation widely applied to domestic jurisdictions. ${ }^{44}$ Contexts of regime complexity, functional decentralisation to private actors and a shift away from directly enforceable regulation have opened up new modalities of governance to a range of non-state actors and institutions. In contrast to hierarchy and delegation, orchestration is not premised on contractual obligation, but rather on voluntary participation within multi-actor systems. It holds when three conditions are met: (1) the orchestrator seeks to influence the behaviour of the target via intermediaries, (2) the orchestrator does not exercise control over the intermediary, which, in turn, (3) cannot compel compliance of the target. In a departure from principal-agent frameworks which

\footnotetext{
40 Johnston suggests that persuasion can be viewed as the 'core' of politics and a vital component of effective governance. lain Johnston, 'Treating International Institutions as Social Environments', International Studies Quarterly 45, no. 4 (2001): 496.

${ }^{41}$ Hafner-Burton, 'A social science of human rights'.

42 Johnston, 'Treating International Institutions as Social Environments', 491.

${ }^{43}$ Abbott et al., International Organizations as Orchestrators.

${ }^{44}$ Kenneth Abbott and Duncan Snidal, 'Strengthening International Regulation Through Transnational New Governance: Overcoming the Orchestration Deficit', Vanderbilt Journal of Transnational Law 42, no. 2 (2009): 501-578.
} 
emphasises direct command and control of the agent, the orchestration model highlights influence exercised indirectly and via non-binding instruments. A burgeoning empirical literature demonstrates that orchestration is widely used by both IGOs and other governance actors in pursuit of governance goals across a range of issue areas, including human rights. $^{45}$

Orchestration is particularly well-suited to theorising the problem structure thrown up by human rights governance. Concretely, the strategic environment of the UN human rights regime confirms the hypotheses advanced by Abbott et al. that IGO-led orchestration is more likely to occur under conditions of: (1) high goal divergence among principals and/or between principals and the agent, (2) capability deficits on the part of the IGO, (3) weak principal oversight and institutional control mechanisms, (4) and availability of third-party intermediaries. Study of the human rights domain also highlights a fifth hypothesis: an expansive and non-restrictive rule framework. We might add that these factors may also be applicable to other actors and levels of analysis, including sub-state agencies operating within domestic political systems. This theoretical toolkit provides a valuable platform to delineate and describe analytically the global human rights governance domain. Importantly, it also opens up inquiry into opportunity space and the ability of strategic actors to leverage a margin of independent action beyond state agreement.

Orchestration overcomes some of the ontological and political limitations of governance frameworks which emphasise cooperation without due regard for the difficulties and normative dilemmas inherent in governing human rights via direct supranational intervention. As illustrated below, an orchestration approach can illuminate the diversity of means, motive and opportunities available to pro-compliance actors seeking to pursue shared governance objectives within a global human rights architecture. In this sense, it serves as a basis for a more grounded explanatory agenda on the transnational factors which shape the efficacy of global governance programmes. Implicit in the conditional factors underpinning IGO-led orchestration is a deeply political project, whereby the relative power of diverse actors (orchestrator, intermediary and, above all, the target), their respective material capabilities, and ideational authority at the local level will have a powerful bearing on outcomes. Orchestration theory offers a window onto an underappreciated mode of human rights governance, but its emphasis on informational advantage, institutional control mechanisms, correlation of goals and complementary capabilities, while highly suggestive, can only provide partial insight into the political contest which informs compliance outcomes.

As such, it is important to also insert power and politics into this framework. The ability of an IGO orchestrator to enlist local pro-compliance actors has potentially significant power implications. However, it does not, by itself, offer a compelling explanatory theory for the success or failure of such governance innovations, which will ultimately be reliant upon the transformation of domestic politics. Actual implementation of human rights standards is contingent on a host of factors, likely requiring a transnational theory of political transformation. ${ }^{46}$ Related to this, the idea of consent-based governance underspecifies the potential for coercion. Orchestrators may not be as reliant upon voluntary consent as the model presupposes, with material and ideational inducements serving also as top-down mechanisms to influence, sanction and potentially exclude local actors whose ideas run counter to prescriptive global governance programmes. As the next section probes further, such considerations provide the fundamental coordinates for deepening understanding of how, when and why orchestrating actors actually generate compliance.

\footnotetext{
${ }^{45}$ See Abbott et al., International Organizations as Orchestrators; Thomas Pegram, 'Global human rights governance and orchestration: national human rights institutions as intermediaries', European Journal of International Relations, forthcoming.

${ }^{46}$ For an instructive discussion on this point see Greg Shaffer, Transnational Legal Ordering and State Change (Cambridge: Cambridge University Press, 2014).
} 


\subsection{Orchestrating chains of governance in torture prevention}

Scholars have produced valuable insight into the origins of the Optional Protocol to the Convention against Torture regime ${ }^{47}$ However, the significance of this new class of treaty regime is still under-theorised and not well understood. Orchestration theory highlights the agentic elements of this unusually articulated architecture, while also making legible the chain of governance relationships from the global to the local. As Figure 1 illustrates, the formal linkages of the OPCAT architecture map relatively straightforwardly onto this analytical framework, allowing for exploration of the articulation of structures and relationships within a unified governance architecture and its effects.

Figure 1: Multi-scale torture prevention governance through orchestration

Model A

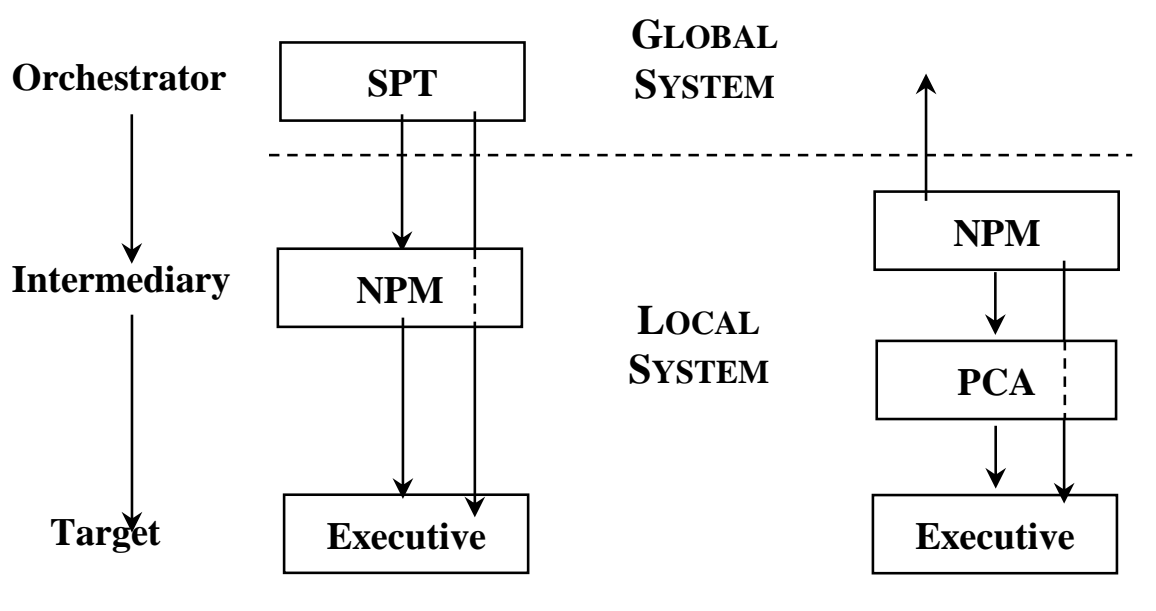

Model B

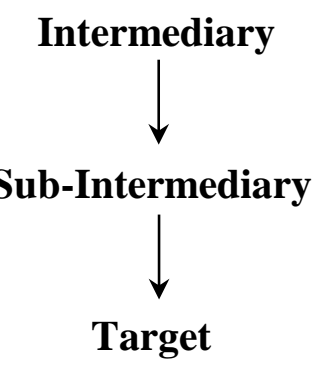

According to model A, the UN Sub-Committee on the Prevention of Torture (SPT) is the Orchestrator seeking to influence the Target: national executives. Bridging the two is the Intermediary: National Preventive Mechanisms (NPMs). Importantly, orchestration theory also allows us to extend the chain of governance to the national and sub-national level, as illustrated in Model B. Here the intermediary also enlists further Sub-Intermediaries or ProCompliance Actors (PCAs), including sub-state and civil society constituents. The model opens up inquiry into alternating roles within the governance architecture, with the intermediary potentially assuming an orchestrator function at the local level. The model also highlights how similar factors may encourage coordinated action along the entire governance chains. As such, orchestration offers the promise of a unified theory of governance.

When applied to the specific issue of torture prevention, orchestration can assist in directing our attention to key structural aspects of the underlying strategic environment. This type of human rights violation displays a particular problem structure, the pathologies of which are likely to have a powerful bearing on any potential solution. The hypotheses advanced by Abbott et al. can serve to explain variations in orchestration in this domain. For example, principal moral hazard is severe, given that torture violations generally implicate either directly or indirectly the state (or state actors) as the responsible party. As such, this type of violation is likely to feature high goal divergence among principals (some of which may credibly commit) as well as between principals and their agent. The high probability of principal moral hazard, counter-intuitively, may also lead to weaker formal oversight as states intentionally insulate the agent in order to enhance credibility. Coupled with the fact

\footnotetext{
${ }^{47}$ Murray et al., The Optional Protocol to the UN Convention Against Torture (Oxford: Oxford University Press, 2011).
} 
that human rights is generally not associated with the 'high politics' of security or trade and therefore not subject to strict intergovernmental management, the conditions for IGO-led orchestration to arise are in place.

If principal moral hazard is a concern at the international level, it becomes acute within domestic political systems where multiple state officials may have a strong interest in evading or obstructing compliance. It is perhaps unsurprising that systematic data on torture incidence is extremely difficult to collect or verify. Given their own capability deficits, IGOs are heavily reliant upon local actors for implementation of a torture prevention programme, as well as assessment of its efficacy. As such, IGOs may achieve efficiency-gains through successful enlistment of intermediaries (Model A). However, as the action moves to the domestic level (Model B), the actual effects of IGO-led orchestration are likely to be highly dependent upon a host of local political conditions. As developed here in relation to the UN torture prevention regime, this may result in outcomes which are starkly different to those predicted in the formal model or those intended by the orchestrator.

\section{A. The Orchestrator}

The SPT is the OPCAT focal actor within UN structures charged with closing the compliance gap between rule-authority and rule-implementation. Nominally a sub-committee to the longstanding Committee Against Torture (CAT) ${ }^{48}$ the SPT forms part of an integrated torture prevention regime but is more accurately understood as a free-standing monitoring body rather than as a subsidiary to the CAT. ${ }^{49}$ The OPCAT seeks to imprint a proscriptive rule framework in a global system of inspection of places of detention coordinated by an international body of independent experts. Establishing no new norms or standards, OPCAT is instead focused on implementing a well-established normative framework prohibiting torture through innovative procedural obligations. ${ }^{50}$ For the architects of OPCAT, the SPT forms the centre-piece of a novel legalisation of human rights architecture which obliges states to recognise the jurisdiction of a global monitoring structure, as well as an analogous domestic structure (Model A, Figure 1).

As a political unit, the SPT comprises 25 independent experts who are appointed by state parties to the Protocol, but serve in their personal capacity for a four-year term, renewable once. ${ }^{51}$ In contrast to other treaty bodies, OPCAT seeks to limit state oversight by explicitly setting down independence and impartiality criteria for membership. ${ }^{52}$ The Convention also requires equitable geographical and gender representation on the subcommittee. ${ }^{53}$ The vast majority of SPT members display significant expertise and are generally viewed as credible. ${ }^{54}$ However, the presence of two current government officials on the SPT is cause for concern. ${ }^{55}$ Perception of independence is vital to the legitimate authority of the SPT. It must guard against actual or perceived instrumentalization by state parties. Countervailing background norms are also apparent in the final OPCAT text which may exert a conservative pull on the appropriate scope of SPT orchestration activities. ${ }^{56}$

\footnotetext{
${ }^{48}$ The CAT is one of ten UN treaty bodies established to supervise state compliance with their obligations under international human rights treaties.

${ }^{49}$ Murray et al. The Optional Protocol, 141.

${ }^{50}$ The general prohibition of torture is one of few state practices outlawed under the norm of jus cogens. Matt Pollard and Nigel Rodley, 'Criminalisation of Torture: State Obligations under the United Nations Convention against Torture', European Human Rights Law Review 11, no. 2 (2006): 115-141.

${ }^{51}$ As of June 2014, OPCAT has only 72 states parties (compared to the Convention which has 155 parties).

52 OPCAT, Article 5(6).

${ }^{53}$ Currently, only forty percent of members are women. All regions are represented.

${ }^{54} \mathrm{CVs}$ available at: http://www.ohchr.org/EN/HRBodies/OPCAT/Pages/Membership.aspx

${ }^{55}$ The representative from Estonia and Macedonia are government employees.

${ }^{56}$ Article 36 of OPCAT requires that SPT members 'refrain from any action or activity incompatible with the impartial and international nature of their duties'.
} 
The SPT self-identifies as 'a new generation of United Nations treaty body with a unique mandate'. ${ }^{57}$ The SPT performs three core tasks, to: (1) conduct country-visits in places of deprivation of liberty, (2) coordinate the work of NPMs, and (3) cooperate with other UN and regional organisations. ${ }^{58}$ Unlike all other treaty bodies, it undertakes field visits rather than considering state reports or individual communications. It therefore proactively engages in information gathering and monitoring activities to arrive at an independent assessment of state compliance with their obligations. In effect, the SPT exercises a police patrol form of accountability mandated to: (1) establish a programme of regular visits, (2) choose the places it wants to visit, and (3) propose follow-up visits. ${ }^{59}$ States are required to receive the SPT and grant it unrestricted access on its territory without prior consent. ${ }^{60}$ This is a seachange from the oversight, subject to state consent, exercised by the CAT inquiry procedure or the UN independent expert or 'special procedure' on torture. ${ }^{61}$ Whereas the authority of the CAT is located mainly in its rule-making function, that of the SPT can be traced to its rule-implementation prerogatives. ${ }^{62}$ As one member puts it:

The promise of the SPT is that it is the largest UN human rights treaty body, which goes to the field and is part of an official system with a constellation of national bodies, given legitimacy by an international treaty. ${ }^{63}$

It is important to note that the SPT is the product of intergovernmental negotiation. This is apparent in a mandate which emphasises cooperation with the target (what Abbott et al. refer to as 'collaboration'). ${ }^{64}$ SPT reports and recommendations based on country visits are intended to inform dialogue with the target, not confrontation. In keeping with UN practice, SPT findings are confidential. However, cooperation on torture prevention confronts the problem of principal moral hazard, with state agencies often implicated in violations. ${ }^{65}$ Given the egregious nature of the crime, state goals often run counter to those of the SPT. The experience of the Committee Against Torture attests to potential challenges. Although empowered, subject to state cooperation, to conduct confidential inquiries into allegations of systematic torture, the CAT has carried out only eight such inquiries. ${ }^{66}$ Between 2007 and 2013, the SPT conducted nineteen country visits, with just ten states consenting to the resulting report being made public. Although the SPT conducts its work in 'a spirit of cooperation' with state authorities, cooperation has its limits.

As such, the SPT has motive to engage in orchestration activities. What of the means and opportunity? As noted above, the SPT forms part of a novel dual-level architecture and is expected to steward system-wide coordination. This is most visible in its formal linkages to National Preventive Mechanisms (NPMs). While this relationship is formalised in the OPCAT architecture and displays shades of delegation, in practice, the SPT has few, if any, instruments with which to directly control the NPM. As such, the efficacy of the arrangement relies largely on goal convergence between both SPT and NPM. ${ }^{67}$ SPT incentives to

\footnotetext{
${ }^{57}$ See SPT, First Annual Report of the SPT, UN Doc. CAT/C/40/2 (2008), para. 5.

${ }^{58}$ Article 11, OPCAT.

${ }^{59}$ See Article 14, OPCAT.

${ }^{60}$ Article 12(a), OPCAT

${ }^{61}$ Special procedures are the mechanisms established by the Human Rights Council to address either specific country situations or thematic issues.

${ }^{62}$ The CAT cannot impose binding obligations, but does issue authoritative interpretations.

${ }^{63}$ Wilder Taylor, SPT Member. Quoted in APT, The Global Forum on the OPCAT (Versoix: APT, 2012), 28.

${ }^{64}$ Abbott et al. International Organizations as Orchestrators, Ch. 1.

65 Darius Rejali, Torture and Democracy (Princeton: Princeton University Press, 2007).

${ }^{66}$ Inquiries have targeted Nepal, Brazil, Serbia and Montenegro, Mexico, Sri Lanka, Peru, Egypt and Turkey.

${ }^{67}$ Article 11(b), OPCAT.
} 
orchestrate are in part functional, a reflection of its own capability deficits. The subcommittee has struggled with a budget which does not reflect the cost of its visitation mandate, coupled with no allocation for NPM-support activities. ${ }^{68}$ Most significantly, the NPM provides the SPT with an additional lever to enhance the legitimate authority of the OPCAT architecture, as well as consistency in application of standards at the local level. It also offers a conduit to engage in orchestration activities with other torture prevention actors, including civil society and the media. Civil society has appealed to the SPT to be more assertive in using its political leverage, especially with regard to designation and strengthening of NPMs. ${ }^{69}$

Enhancing informal spaces for dialogue with civil society and potential sub-intermediaries is important if the SPT is to establish a stable orchestration arrangement. The inaccessibility of confidential Geneva-based SPT sessions has been criticised. In terms of advancing compliance, public scrutiny of SPT decision-making by third parties may be vital to constructing a base for collective political action against often powerful opposing domestic forces, and to ensure local ownership of governance rule systems at the societal level. Emergent efforts in this direction are apparent, with greater transparency on in-country mission schedules and SPT meetings. Notably, the SPT has also recently launched a Special Fund to help finance local implementation of its recommendations. States, NPMs and other sub-state actors and NGOs are eligible to apply for funding 'if the proposed projects are to be implemented in cooperation with eligible States parties and/or NPMs' ${ }^{70}$ The SPT convenes an OPCAT Contact Group comprising eleven organisations with global expertise and presence in the field of torture prevention. ${ }^{71}$ Grassroots organisations and NPMs are, however, absent from this network. One observer notes that:

'In the final [OPCAT] text, the role of the Subcommittee is projected as being that of general oversight, exercising something of a paternalistic interest in the operation and functioning of the [NPMs]'.

The SPT membership has shown itself willing to make modest moves away from a 'paternalistic' mode of governance towards a more horizontal and deliberative decisionmaking model conducive to system-wide orchestration. More could be done. Its performance, and the effect of the OPCAT regime on closing compliance gaps, may depend upon it.

\section{B. The Intermediary}

The NPM forms the second core pillar of the OPCAT architecture; the formal linkage of a global monitoring body with an equivalent domestic structure. The legal coupling of global and domestic mirror structures described here is a significant departure from treaty law precedent, with NPMs offering a gateway for installing rule systems across jurisdictions. It is important to note that states are required to 'maintain, designate or establish' an NPM within one year of OPCAT ratification and therefore do not necessarily need to create a new structure. ${ }^{72}$ Thus far, fifty-two (seventy-two percent) of state parties to the Protocol have

\footnotetext{
${ }^{68}$ The annual budget for servicing all ten treaty bodies in 2012 was near $\$ 56$ million.

69 "It is high time to explode the myth that the SPT should only interact with NPMs and States parties through formal in-country missions". Mark Thompson, APT Secretary General. Quoted in APT, The Global Forum on the OPCAT (Versoix: APT, 2012), 28.

${ }^{69}$ Abbott et al. International Organizations as Orchestrators, Ch. 1.

${ }^{70}$ See http://www.ohchr.org/EN/HRBodies/OPCAT/Pages/SpecialFund2014.aspx (Accessed 29 June 2014)

${ }^{71}$ See http://www.ohchr.org/EN/HRBodies/OPCAT/Pages/OPCATContactGroup.aspx (Accessed 29 June 2014).

${ }^{72}$ Article 17, OPCAT
} 
designated an NPM. ${ }^{73}$ Of those fifty-two NPMs, the majority are pre-existing standalone ombudsmen and national human rights institutions (NHRIs) offices. ${ }^{74} \mathrm{~A}$ minority of states have established multi-institution NPMs ${ }^{75}$ or purpose-built new mechanisms. ${ }^{76}$

NPMs possess a range of governance capabilities at the domestic level which make them desirable intermediaries. They draw legitimacy from their standing as a national-level state body codified in law. Their role in receiving individual complaints, monitoring domestic torture prevention and follow-up with SPT recommendations is of particular note. ${ }^{77}$ NPMs also have direct access to the target constituency: government officials. Such access may yield actionable information for the SPT when seeking to engage pro-compliance sub-state actors or, conversely, bypass obstructive veto players. In sum, an effective NPM may enhance the SPT's ability to not only advance compliance with torture prevention standards, but also advance compliance through the socialization of domestic actors. For their part, orchestration with the SPT offers NPMs access material and ideational resources. Endorsement by the SPT may yield significant domestic benefits, legitimising the activities of the NPM, encouraging sub-intermediary orchestration, and elevating the NHRI as a national focal actor.

Although generally lacking enforcement powers, many NPMs are vested with significant protection prerogatives, including legal petition powers, ex officio powers of investigation, and the ability to censure uncooperative state officials. OPCAT obliges a series of NPM operational features, including provisions on unrestricted access to all places of detention, subpoena powers, and engagement with the SPT. ${ }^{78}$ NPMs can also engage in awarenessraising and promotional activities, including advising on legislation, media interventions and joint-campaigns with local stakeholders, serving as both venues and agents for mobilization. This stands in stark contrast to the confidentiality and impartiality restrictions imposed upon the work of the SPT. The task for the SPT and other torture prevention stakeholders is how to ensure that NPMs are actually enabled to fulfil their mandate.

A central concern is independence. Given their location in domestic political processes, NPMs are highly sensitive to the exercise of power within domestic political systems. Article 18 of OPCAT obliges states to guarantee the 'functional independence' of the NPM. States are further directed to the Paris Principles - UN endorsed guidelines on NHRI independence, including a prohibition on government representation. ${ }^{79}$ Resistance to the NPM proposal during OPCAT largely stemmed from doubts over the efficacy of national institutions. ${ }^{80}$ NPMs are an example of dual delegation, deriving authority from their formal designation by both state and IGO structures. However, dual delegation does not equate to parity of principal control. Notably, a number of SPT members are also members of NPMs with potentially important implications for governance relationships within this dual delegation arrangement. ${ }^{81} \mathrm{~A}$ key challenge for the NPM therefore reflects a unique situation within the OPCAT architecture: how to balance independence with accountability to multiple

\footnotetext{
${ }^{73}$ Only two states have made a declaration to postpone implementation of the NPM for up to five years.

${ }_{4}$ NHRIs are state-funded, but formally independent, human rights agencies, enacted by constitutional amendment or legislation, generally appointed by the legislature, and composed of representatives with human rights expertise, including civil society.

${ }_{75}$ See Argentina and Macedonia for instance.

${ }_{77}^{76}$ Honduras established a new National Committee for the Prevention of Torture.

${ }^{77}$ OPCAT directs the SPT to communicate its recommendations to the NPM without prior state consultation or consent. Article 16, OPCAT

${ }^{78}$ Article 20, OPCAT

${ }^{79}$ See Principles Relating to the Status and Functioning of National Institutions, UN General Assembly, Res. 48/134, 20 December 1993.

${ }^{80}$ Murray et al., The Optional Protocol, 44.

${ }^{81}$ The SPT members from Moldova and Serbia are also affiliated to the NPM.
} 
sites of authority (IGO, state and civil society). This is readily apparent in highly political processes of NPM designation, with many subject to protracted delays. The SPT has begun to take a more concerted interest in domestic NPM establishment processes, publicising best practice. ${ }^{82}$

Orchestration theory provides a valuable toolkit for disaggregating governance domains and establishing the conditions under which diverse actors will orchestrate action as an adaptive response to their strategic environments. However, what can it tell us about the outcome of orchestration efforts? As this discussion highlights, torture prevention agents (IGOs, NPMs, and other PCAs) have varying degrees of freedom to pursue their governance objectives. In some circumstances, the principal (states) may also credibility commit to the global governance programme. In many instances this is not the case. The powerful intervening effects of domestic political contestation are implicit in the notion of bypassing the principal via an intermediary. There is always a politics to institutional outcomes, with variable degrees of contestation at different scales of governance.

A focus on goal convergence across political units along governance chains as proposed by orchestration theory is highly instructive. Focusing on goal convergence between orchestrator and intermediary, we can construct a set of four orchestration outcomes (Figure 2). As such, orchestration theory provides for alternative outcomes. A pending task is to explicitly theorise the observed deviation from the linear model. When are we more likely to observe cascade, reverse or competitive orchestration? Even where a simple model of orchestration takes hold, what is the effect of this governance configuration on closing the compliance gap? NPMs may engage in a range of socialization activities (training of state officials, educational campaigns, agenda-setting for instance), but under what conditions would we expect such activities to actually generate compliance? A theoretically-informed literature in comparative politics offers some clues as to where answers to such questions may lie. ${ }^{83}$

Figure 2: A four-fold typology of orchestration outcomes ${ }^{84}$

\begin{tabular}{|cccc|}
$\begin{array}{c}\text { Simple } \\
\text { Orchestration }\end{array}$ & $\begin{array}{c}\text { Competitive } \\
\text { Orchestration }\end{array}$ & $\begin{array}{c}\text { Cascade } \\
\text { Orchestration }\end{array}$ & $\begin{array}{c}\text { Reverse } \\
\text { Orchestration } \\
\text { Orchestrator }\end{array}$ \\
$\begin{array}{c}\text { Orchestrator } \\
\downarrow\end{array}$ & Orchestrator & Orchestrator \\
Intermediary & Intermediary & Intermediary & Intermediary \\
$\downarrow$ & $\Varangle$ & $\downarrow \downarrow \downarrow$ & $\uparrow$ \\
Target & $\Varangle$ & Target & Target \\
& Target & & \\
\hline
\end{tabular}

\footnotetext{
${ }^{82}$ See Report on the visit made by the SPT for the purpose of providing advisory assistance to the NPM of Moldova, UN Doc. CAT/OP/MDA/R.1, 9 January 2013.

${ }^{83}$ See, for example, Garry Rodan and Caroline Hughes, The Politics of Accountability in Southeast Asia (Oxford: Oxford University Press, 2014).

${ }^{84}$ This typology is inevitably highly stylized but will serve as a useful departure point for further problematisation through empirical application.
} 
Figure 2 provides a framework to begin to probe the implications of orchestration arrangements for understanding the actual efficacy of global governance programmes. The OPCAT regime is a particularly instructive domain, given its unusually formalised multi-level governance rule system as well as its emphasis on goal convergence among key political units. Simple orchestration conforms to the formal linear prediction of the model, with the NPM serving as intermediary to the SPT and the OPCAT implementation programme. Simple orchestration relies upon goal convergence between the Orchestrator and Intermediary, with the Orchestrator exercising no hierarchical control. The NPM also serves to mediate the deep governance level along a governance chain extending from the SPT to the sub-local level (Model B, Figure 1). This dispersal of delegated authority from an IGO to a national mechanism can empower the NPM to assume an orchestrator role in its own right. Indeed, NPMs are generally exhorted to be 'a leader' in torture prevention. ${ }^{85}$

Voluntary participation of NPMs and other PCAs is predicated on the material and ideational support of the SPT. However, in practice this may not be sufficient to ensure goal convergence. Ensuring mutual benefit is therefore central to the orchestration equilibrium. Absent mutual benefit in a resource finite-environment, the SPT and NPM may conceivably enter a situation of competitive orchestration whereby both vie for resources (both material and political capital). As noted above, however, political contestation is implicit in the orchestration enterprise. This is particularly likely to be the case at the domestic level where compliance efforts often confront powerful institutional, political and social counterpressures. In turn, intermediaries and other local actors are responsive to different political stimuli, and may pursue agendas independent of the OPCAT rule system. This does not necessarily presuppose goal divergence and orchestration failure, but it does complicate the linear relational model and indicate potential changes in the causal direction of the governance chain.

However, it is also conceivable that an orchestration arrangement could result in a complete reversal of the causal chain. The intermediary role of an NPM is conditional upon the complicated task of establishing working relationships with diverse actors within and outside state structures. NPMs are vulnerable to government capture, especially where torture violations implicate powerful state officials. Such an outcome is a threat to the efficacy of the OPCAT regime, with intermediary preferences aligning with the target to influence the IGO in, effect, a reverse-orchestration. Evidence points to some national institutions successfully protecting human rights, with others having effectively undermined them on behalf of government. ${ }^{86}$ Observers concur that individual leadership is crucial. As a former UN special procedure comments: "With a mandate like torture, it goes to the very heart of the regime. A [national institution] that feels at all insecure is unlikely to raise its voice on these issues" ${ }^{87}$ Importantly, such an outcome does not necessarily spell the end of the formal orchestration arrangement. In a worst case scenario, the target is able to evade and deflect scrutiny through effective management of the orchestrator by proxy. In this instance, an orchestration arrangement may persist despite impeding domestic compliance with the OPCAT rule system.

Inclusion within the formalised OPCAT architecture may offer NPMs additional insulation from political interference. However, the ability of the SPT to intervene on dubious NPM selection processes is complicated by a commitment to collaboration rather than condemnation. ${ }^{88}$ Condemnatory duties may therefore fall upon other stakeholders within civil

\footnotetext{
${ }^{85}$ APT, The Global Forum on the OPCAT, 33.

${ }^{86}$ For in depth analysis of NHRIs as human rights actors see Ryan Goodman and Thomas Pegram, (eds.), Human Rights, State Compliance, and Social Change (New York: Cambridge University Press, 2012).

${ }^{87}$ Confidential interview by author with former UN special procedure, 16 December 2009.

${ }^{88}$ See http://www.ohchr.org/EN/HRBodies/OPCAT/Pages/OPCATIntro.aspx (Accessed 29/06/14).
} 
society. Civil society has played a prominent role in the development of the OPCAT project, although it does not feature prominently in the OPCAT text. The presence of subintermediaries as depicted in Model B may be a crucial and under-specified corollary to the efficacy of the OPCAT regime. The impact of national level political contestation among competing social forces upon the efficacy of global governance programmes requires explicit theorising, a task which exceeds the explanatory scope of orchestration theory as it is currently conceived. This auxiliary support from NGOs and other societal actors is likely to be particularly important in contexts where formal compliance remains partial and OPCAT standards are subject to contestation. They can serve as powerful interlocutors in translating the OPCAT basic operational norms into local 'vernacular'. ${ }^{89}$ In a scenario of cascade orchestration as depicted in Figure 2, motivated local stakeholders not formally integrated into the OPCAT architecture may nevertheless advance goals consistent with its rule system, but absent formalised relations with the orchestrator.

\section{Conclusion}

The notion of architecture provides a valuable way into identifying not only what is actually global in global human rights governance, but also how the global is connected to the local. Exploring the Optional Protocol has demonstrated how imprinting a global governance rule system in both structure and governance relationships can begin to bridge the steps which separate international instruments from practices on the ground. This study highlights the articulation of a global human rights architecture in substantive obligations, as well as, and most radically, a series of procedural mechanisms which underpin a novel two-tier implementation apparatus. However, as this study explores, the existence of an orchestration arrangement does not necessarily translate into an effective mode of governance. The OPCAT architecture aspires to globality, even if many states are still to ratify the instrument. Not only does it assume structural form above the level of the nation state, political units within the architecture display significant autonomy and power and there is significant unbundling of functions among interacting actors across levels.

This article has sought to highlight opportunity spaces for purposive action within the OPCAT architecture. Orchestration theory as developed by Abbott and colleagues has served to frame the OPCAT strategic environment. Descriptively, orchestration serves to clearly specify governance actors, with particular attention to questions of their location, focality, authority and resources. Analytically, it asserts agency and process as important corollaries to structural explanations for governance outcomes. It identifies new authorising structures not only within IGO settings, but also extending to domestic political systems. Different from orthodox modes of governance which assume hierarchical control or the direct interface between principal and agent, orchestration in emphasising indirect channels of influence via third party intermediaries offers a useful addition to modes of human rights governance. In turn, orchestration offers a unified theory of governance - one capable of engaging with both a transnational and domestic politics of governance beyond command and control relationships.

Orchestration also provides a basis for advancing an explanatory agenda on the efficacy of global governance programmes. As indicated in this study, a crucial condition for orchestration to occur is limited state control and oversight of the agent. Clearly, this is particularly important in a regulatory domain such as torture prevention which displays severe principal moral hazard - but is also likely to be applicable to other issues areas. The implication that how political units are connected to centres of state authority matters provides the fundamental coordinates for deepening transnational theorising around compliance. State authority remains a prominent influence on the politics of compliance and a core consideration as the focus turns away from the articulation of formal structures to

\footnotetext{
${ }^{89}$ Sally Engle Merry, Human Rights and Gender Violence (Chicago: University of Chicago Press, 2006).
} 
questions of efficacy. When and why do global structures matter in tilting domestic political contests in favour of local pro-compliance coalitions? Orchestration allows us to hypothesis four possible outcomes of such an arrangement, with important implications for domestic compliance politics. By specifying goal convergence and divergence between the SPT and NPMs, the study highlights the indeterminacy of orchestrated modes of governance and the potential for unintended consequences. This exploratory mapping of admittedly highly stylised relations will be further problematised in a follow-on empirical project on OPCAT implementation.

The installation of formal structures at the domestic level carries the promise of imprinting global standards onto domestic practice. However, function often does not follow form. This is a realm not simply of interlocking structure to use the rationalist parlance, but also governance relationships. Goal convergence and preference ordering - both ideational and material - and the ability of steward actors to generate broad based consensus around a global governance programme is likely to be crucial to the stability, durability, and performance of the OPCAT architecture. In other words, global governance as process requires careful attention to actors' motives, not only means and opportunity. But perhaps the crucial question turns on explaining how these actors actually operate in practice and when and why they matter for compliance politics. Some observers have concluded that the inter-state legal human rights system is in terminal decline, declaring 'the endtimes of human rights'. ${ }^{90}$ However, viewed from another vantage point, such claims may under-estimate the distributional power implications, especially once these structures are articulated into domestic politics.

${ }^{90}$ Stephen Hopgood, The Endtimes of Human Rights (Ithaca: Cornell University Press, 2014). 